\title{
Numerical Simulation of Cropping
}

\section{Citation}

Tvergaard, Viggo, and John W. Hutchinson. 2014. "Numerical Simulation of Cropping." J. Appl. Mech. 81 (7) (March 6): 071002. doi:10.1115/1.4026891.

\section{Published Version}

doi:10.1115/1.4026891

\section{Permanent link}

http://nrs.harvard.edu/urn-3:HUL.InstRepos:14023017

\section{Terms of Use}

This article was downloaded from Harvard University's DASH repository, and is made available under the terms and conditions applicable to Other Posted Material, as set forth at http:// nrs.harvard.edu/urn-3:HUL.InstRepos:dash.current.terms-of-use\#LAA

\section{Share Your Story}

The Harvard community has made this article openly available.

Please share how this access benefits you. Submit a story.

\section{Accessibility}




\title{
J. Appl. Mechanics 2014 JAM DOI:10.1115/1.4026891
}

\section{Numerical simulation of cropping}

\author{
Viggo Tvergaard \\ Department of Mechanical Engineering, Technical University of Denmark \\ John W. Hutchinson \\ School of Engineering and Applied Sciences, Harvard University
}

\begin{abstract}
Cropping is a cutting process whereby opposing aligned blades create a shearing failure by exerting opposing forces normal to the surfaces of a metal sheet or plate. Building on recent efforts to quantify cropping, this paper formulates a plane strain elastic-plastic model of a plate subject to shearing action by opposing rigid platens. Shear failure at the local level is modeled by a cohesive zone characterized by the peak shear traction and the energy dissipated by shear failure process at the microscopic level. The model reveals the interplay between shear cracking and the extensive plastic shearing accompanying the cutting process. Specifically, it provides insight into the influence of the material's microscopic shear strength and toughness on the total work of cropping. The computational model does not account for deformation of the cropping tool, friction between sliding surfaces and material temperature and rate-dependence.
\end{abstract}

Key words: Cropping, shear fracture, finite strain plasticity, cohesive zone, fracture energy

\section{Backround to the Present Study}

Cutting by forced shear-off, often called blanking or cropping, is widely employed to cut flat sheets and plates of structural metals having thickness ranging from sub-millimeter to ten millimeters or more. Forced shear-off can also occur in the plugging process in ballistic penetration when a high velocity projectile impacts a plate. The simplest notions of cropping imagine that the process is essentially a shear failure within a localized shear band that is primarily controlled by the shear strength of the material. The present paper continues the line of investigation initiated in a series of papers by Atkins [1-4] who takes the view that shear cracking and material shear toughness play an essential role in the cropping process and in determining the energy required for cropping. The series of Atkins' papers presents models of cropping with an increasing level of sophistication without recourse to finite element models of finite plastic straining. An early paper by Zhou and Wierzbicki [5] also employs analytical 
modeling and accounts for tensile fracture as well as shearing in the cropping process, which in their paper and in some of the earlier literature is referred to as blanking.

Following Atkins, the view adopted in this paper is that cropping is a large scale yielding fracture problem dominated by shear. As in the case of the present authors' earlier work on elastic-plastic crack growth in mode I [6] and mixed mode [7], the present paper exploits the finite element analysis to deal with large plastic strains. The present paper also makes use of recent developments in modeling fracture under shear dominated conditions. The cropping model developed here imbeds a cohesive shearing zone, which, in a phenomenological manner, represents the microscopic shear localization and fracture processes, within a finite element model that accounts for the geometric distribution of the large plastic shear strains that occur in the cropping process. The model quantifies the interplay between the microscopic shearing failure process and the extensive macroscopic plastic shearing. A systematic study is made of the effects of the microscopic fracture energy and the material stress-strain properties on the cropping force-displacement behavior and the macroscopic cropping energy.

Alternative approaches to studying cropping could be based on analyses that adopt either a critical effective plastic strain as a failure criterion or a constitutive law that incorporates damage and a failure criterion. When carefully calibrated, the critical plastic strain criterion has proved effective in the studies of ballistic plugging of plates by projectiles, e.g., Borvik, et al. [8,9] and Nahshon, et al [10]. Xue, et al. [11] demonstrated the applicability to ballistic shear-off of the Gurson model $[12,13]$ of void-based damage and failure, extended to account for damage in shear. A distinct advantage of the cohesive zone model adopted in the present paper is that the microscopic strength and fracture energy are well defined parameters and their role in establishing macroscopic behavior clearly emerges.

1.1 An Illustrative Cropping Experiment. Xue, et al. [11] carried out carefully designed cropping tests on plates of the steel, DH 36, of thickness $h=3 \mathrm{~mm}$. As illustrated in Fig. 1, the tests provided the response of a hard tool steel cylindrical plunger pushing against a plate clamped tightly outside the plunger radius by a stiff fixture. The nominal shear stress supported by the plate at radius $R, P /(2 \pi R h)$, is plotted as a function of the plunger displacement divided by the plate thickness, $\Delta / h$, for one test in Fig. 1b. The peak nominal shear stress is about $420 \mathrm{MPa}$ at $\Delta / h \cong 0.25$. The largest plunger displacement shown is $\Delta / h \cong 0.55$. The load 
drops precipitously at larger displacements such that the macroscopic work of cropping is directly related to the area under the curve in Fig. 1b. Tests were interrupted at various stages and cross-sections of the sheared-off region were opened to viewing by electrodischarge machining (EDM). In addition to intense plastic shearing throughout the region of shear-off, shear cracks are observed. Examples of shear cracks near the corner of the plunger at $\Delta / h=0.5$ are seen in Fig. 1d, where is noted that some cracks form slightly offset from the line extending the corner of the plunger.

The radius of the plunger in the Xue, et al. [11] test is sufficiently large compared to the plate thickness, i.e., $R / h=6.3$, such that it is reasonable to assume that the specific cropping work, $\Gamma_{C R O P}$, with units work/area cut, would be nearly the same as that measured for a long straight cut. The total work done by the plunger in Fig. $1 \mathrm{~b}$ is $W=\alpha 2 \pi R h^{2}\left(\bar{\tau}_{Y}\right)_{\text {MAX }}$ where $\left(\bar{\tau}_{Y}\right)_{M A X}$ is the maximum nominal shear stress and $\alpha$ is a dimensionless numerical factor that depends on the parameters of the system. The specific cropping work (per area cut) is

$$
\Gamma_{C R O P}=\frac{W}{2 \pi R h}=\alpha\left(\bar{\tau}_{Y}\right)_{M A X} h
$$

For the $3 \mathrm{~mm}$ thick plate of DH 36 in Fig. $1 \mathrm{~b},\left(\bar{\tau}_{Y}\right)_{M A X}=420 M P a$ and the area under the loaddisplacement curve implies $\alpha \cong 1 / 2$, such that $\Gamma_{C R O P} \cong 6 \times 10^{5} \mathrm{Jm}^{-2}$. This value is consistent with the specific work of cropping reported for other metals in the early work of Johnson and Slater [14] discussed in [4,5].

One of the aims of this paper is to relate the specific macroscopic work of cropping, $\Gamma_{\text {CROP }}$, to the specific microscopic work of shear fracture of the material, $\Gamma_{0}$, both of which are measured in units of energy/area, $\mathrm{Jm}^{-2}$. Fig. 2 presents simulations based on the extended Gurson model [13] for the shear stress strain behavior accounting for damage in the form of voids with an initial effective volume fraction, $f_{0}$. A pure power-law material is used to describe the undamaged material in the Gurson model with a tensile relation between the true stress and logarithmic strain given by $\sigma=\sigma_{R} \varepsilon^{N}$. The extended model accounts for progressive shear damage, due to shear distortion of the voids, in a phenomenological manner such that in 
shear (with zero stress triaxiality) damage grows according to $\dot{f}=k_{\omega} f \dot{\gamma}$ with $\gamma$ as the logarithmic shear strain and $k_{\omega}$ as the shear damage coefficient $[13,15,16]$. The trends in Figs. 2a and $\mathrm{b}$ should only be regarded as qualitative since no effort has been made to include the final coalescence stage of the shear failure process. Moreover, microscopic mechanisms other than the void-mechanism may be responsible for softening and shear localization. Nevertheless, the results illustrate the roles of damage, $f_{0}$, and damage growth susceptibility as measured by $k_{\omega}$ in determining the peak shear stress at which point shear localization would begin. This stressstrain behavior has been used to compute the work dissipated in shearing subsequent to attainment of the peak shear stress in a shear band of thickness $D$. Fig. 2c presents the specific work dissipated in the shear band per area, $\Gamma_{0}$, normalized by $\sigma_{R} D$, as a function of the shear damage coefficient for several values of the initial damage. We emphasize that the purpose of Fig. 2 is not to present quantitatively reliable predictions for the shear response or the work dissipated in a shear band but, rather, to suggest trends and to provide rough estimates.

Fig. 2c indicates that the specific work of shear fracture scales as

$$
\Gamma_{0}=\beta \sigma_{R} D
$$

where $\beta$ is a factor of order unity depending on the initial damage level and the susceptibility of the damage to shearing as modeled here by the coefficient $k_{\omega}$. For materials failing by the mechanism of void nucleation, shear distortion and coalescence, the thickness, $D$, of the shear localization zone before the final coalescence stage is usually on the order of the spacing between the dominant voids. Thus, for many ductile alloys, $D$ is typically measured in tens of microns. Assume this is so for $\mathrm{DH} \mathrm{36}$, and note also that for this material the tensile stress at a $\log$ strain extrapolated to unity is $\sigma_{R} \approx 1000 M P a$. With $\beta=2$ in (2), this implies for DH 36 that $\Gamma_{0}$ is estimated to lie within the range: $2 \times 10^{4} \mathrm{Jm}^{-2}$ (for $D=10 \mu \mathrm{m}$ ) to $1 \times 10^{5} \mathrm{Jm}^{-2}$ (for $D=50 \mu \mathrm{m})$. The specific macroscopic work of cropping (1) for the $3 \mathrm{~mm}$ plate of $\mathrm{DH} 36$ was estimated to be $\Gamma_{C R O P} \cong 6 \times 10^{5} \mathrm{Jm}^{-2}$. This comparison suggests that the work of cropping is at least 6 times, and possibly as much at 30 times, the specific microscopic work of shear fracture for DH 36. If this comparison is correct, it suggests that the major part of the work of cropping 
is due to plastic deformation occurring outside the shear fracture zone. This relation between the microscopic and macroscopic fracture work is similar to that in mode I crack advance in tough ductile alloys where the macroscopic work of fracture far exceeds the microscopic work of fracture due to plastic dissipation occurring outside the fracture process zone [6]. Nevertheless, in Mode I cracking the microscopic work of fracture remains essential in determining the macroscopic toughness, and it will be seen that a similar dependency holds for cropping.

\section{Definition of the Model and Dimensionless Parameters}

To set the stage for the computational results presented in subsequent sections, the material and geometric parameters governing the cropping model used in this paper will now be listed and the important dimensionless collections of these parameters will be identified. The plate being cut is taken to be isotropic with Young's modulus, E, Poisson's ratio, v, initial tensile yield stress, $\sigma_{Y}$, initial shear yield stress, $\tau_{Y}=\sigma_{Y} / \sqrt{3}$, and strain hardening exponent, $N$. In this paper, no attempt will be made to account for temperature or rate effects in cropping. The process is modeled as quasi-static and the plate material is taken to be rate-independent, excluding any direct relevance to cropping at high temperatures. Elasticity of the cropping tool is also neglected in this study-the surfaces of the cutting tool are taken to be non-deforming. As depicted in Figs. 1a and 3b, the thickness of the plate is $h$ and the gap between the surfaces of the cropping tool is $d$. The band of localized shear and shear fracture will be modeled by a cohesive zone whose primary parameters are the peak shear stress, $\hat{\tau}$, and the specific work of fracture in shear, $\Gamma_{0}$. Full details of the cohesive zone will be given in the next section, including the maximum shearing displacement across the zone prior to loss of shear strength. The maximum shearing displacement can be expressed in terms of $\hat{\tau}$ and $\Gamma_{0}$, which are preferred for specifying the cohesive law in the present study.

It is useful to define the following material reference length,

$$
R_{S}=\frac{1}{\pi\left(1-v^{2}\right)} \frac{E \Gamma_{0}}{\tau_{Y}{ }^{2}},
$$

which is can be interpreted as the extent of the plastic zone ahead of a mode II (shear) crack in plane strain small scale yielding when subject to the mode II stress intensity factor 
$K_{I I}=\sqrt{E \Gamma_{0} /\left(1-v^{2}\right)}$. The only independent material-based length parameter in the present study is $R_{S}$. It is important to appreciate that $R_{S}$ is not the plastic zone size of a mode II crack with specific microscopic work of fracture $\Gamma_{0}$ - that plastic zone size would generally be much larger, i.e., given by (3) with the specific macroscopic work of mode II fracture, $\Gamma_{\text {II }}$, replacing $\Gamma_{0}$. In earlier work on mode I cracking, Tvergaard and Hutchinson [6] introduced the corresponding length for a tensile crack, $R_{0}=\left(1 /\left(3 \pi\left(1-v^{2}\right)\right) E \Gamma_{0} / \sigma_{Y}{ }^{2}\right.$, which is $R_{S} / 9$. With $\Gamma_{C R O P}$ as the specific work expended by the cropping plunger (per area of plate cut) for long straight cuts, two dimensionless forms for the specific cropping work in terms of the parameters identified above will be considered:

$$
\begin{aligned}
& \frac{\Gamma_{C R O P}}{\tau_{Y} h}=g_{1}\left(\frac{h}{R_{S}}, \frac{\hat{\tau}}{\tau_{Y}}, N, \frac{d}{h}, \frac{\tau_{Y}}{E}\right) \\
& \frac{\Gamma_{C R O P}}{\Gamma_{0}}=g_{2}\left(\frac{h}{R_{S}}, \frac{\hat{\tau}}{\tau_{Y}}, N, \frac{d}{h}, \frac{\tau_{Y}}{E}\right)
\end{aligned}
$$

where the dependence on $v$ has not been noted explicitly, and

$$
g_{1}=\frac{\Gamma_{0}}{\tau_{Y} h} g_{2}=\pi\left(1-v^{2}\right) \frac{\tau_{Y}}{E} \frac{R_{S}}{h} g_{2}
$$

Both normalizations will be used to reveal important aspects of the parametric dependencies. For example, it will be seen that the dependence on $\tau_{Y}$ appears mainly through the first two dimensionless parameters in $g_{2}$ in (5) and not through the fifth parameter, $\tau_{Y} / E$, while $g_{1}$ in (4) has a strong dependence on $\tau_{Y} / E$ in its list of parameters.

The preferred dimensionless relation relating the cropping force per length, $F$ with units $\mathrm{Nm}^{-1}=\mathrm{Jm}^{-2}$, and the displacement of the cropping tool through which it works, $\Delta$, is

$$
\frac{F}{\tau_{Y} h}=g_{3}\left(\frac{\Delta}{h}, \frac{h}{R_{S}}, \frac{\hat{\tau}}{\tau_{Y}}, N, \frac{d}{h}, \frac{\tau_{Y}}{E}\right)
$$


By (4), one notes that $g_{1}=\int_{0}^{1} g_{3} d(\Delta / h)$.

\section{Prescription of the Computational Model}

3.1 The Cohesive Zone. As noted above, a cohesive zone model is used to characterize the strength and fracture interface of the plane along which cutting occurs. Because the cutting process is not strictly shearing, a mixed mode traction-separation law is employed in the form introduced in [7] for studying mixed mode interface crack growth. While the tangential displacement, $\delta_{t}$, across the failure plane is expected to dominate the cutting behavior, the model also accounts for a normal separation, $\delta_{n}$. Denote the critical value of $\delta_{t}$ at which the traction vanishes under strictly shearing as $\delta_{t}^{c}$ and similarly denote the critical strictly normal separation by $\delta_{n}^{c}$. Under mixed mode conditions, the displacement measure, $\lambda=\left[\left(\delta_{n} / \delta_{n}^{c}\right)^{2}+\left(\delta_{t} / \delta_{t}^{c}\right)^{2}\right]^{1 / 2}$, is employed such that the tractions drop to zero at $\lambda=1$. The tractions are derived from a potential function given by

$$
\Phi\left(\delta_{n}, \delta_{t}\right)=\delta_{t}^{c} \int_{0}^{\lambda} \tau\left(\lambda^{\prime}\right) d \lambda^{\prime}
$$

where $\tau(\lambda)$ characterizes the traction-separation relation in shear. Use of the potential results in a work of cohesive failure that is the same for all mixed mode separations which is given by (8)

with $\lambda=1$, i.e., $\Gamma_{0}=\delta_{t}^{c} \int_{0}^{1} \tau\left(\lambda^{\prime}\right) d \lambda^{\prime}$. The normal and tangential components of the traction acting on the cohesive failure plane are given by

$$
T_{n}=\frac{\partial \Phi}{\partial \delta_{n}}=\frac{\tau(\lambda)}{\lambda} \frac{\delta_{n}}{\delta_{n}^{c}} \frac{\delta_{t}^{c}}{\delta_{n}^{c}} \quad, \quad T_{t}=\frac{\partial \Phi}{\partial \delta_{t}}=\frac{\tau(\lambda)}{\lambda} \frac{\delta_{t}}{\delta_{t}^{c}}
$$

In this paper the following piecewise linear traction-separation law is used (see Fig. 3a):

$$
\begin{array}{ll}
\tau(\lambda)=\frac{\lambda}{\lambda_{1}} \hat{\tau} & \text { for } 0 \leq \lambda<\lambda_{1} \\
\tau(\lambda)=\hat{\tau} & \text { for } \quad \lambda_{1} \leq \lambda<\lambda_{2}
\end{array}
$$




$$
\tau(\lambda)=\frac{1-\lambda}{1-\lambda_{2}} \hat{\tau} \text { for } \lambda_{2} \leq \lambda<1
$$

Here, $\hat{\tau}$ denotes the maximum shear traction sustained by the failure plane under a mode II shear, i.e., $\delta_{n}=0$. The peak normal traction under mode I separation is $\hat{\sigma}=\left(\delta_{t}^{c} / \delta_{n}^{c}\right) \hat{\tau}$. The specific work of separation per unit area of interface is

$$
\Gamma_{0}=\frac{1}{2} \hat{\tau} \delta_{t}^{c}\left(1-\lambda_{1}+\lambda_{2}\right)
$$

In all the simulations carried out in this paper, the values, $\lambda_{1}=0.15$ and $\lambda_{2}=0.5$, have been used such that $\Gamma_{0}=0.675 \hat{\tau} \delta_{t}^{c}$. Studies of mode I and mixed mode crack growth in elastic-plastic solids indicated that the detailed shape of the traction-separation law as determined by $\lambda_{1}$ and $\lambda_{2}$ are less important than $\Gamma_{0}$ and $\hat{\tau}[6,7]$, and this is expected to be true for cropping as well.

3.2 The Elastic-plastic Constitutive Behavior and the Finite Element Model. The cropped material is elastic-plastic, with the elastic modulus and Poisson's ratio, $E$ and $v$, uniaxial yield stress $\sigma_{Y}$, and strain hardening exponent $N$. This material is described by a finite strain generalization of $J_{2}$-flow theory [17], with the uniaxial true stress-natural strain curve represented by a piecewise power law

$$
\varepsilon= \begin{cases}\frac{\sigma}{E} & \sigma \leq \sigma_{Y} \\ \frac{\sigma_{Y}}{E}\left(\frac{\sigma}{\sigma_{Y}}\right)^{1 / N} & \sigma>\sigma_{Y}\end{cases}
$$

A Lagrangian convected coordinate formulation of the field equations is used for the analyses, with a material point identified by the coordinates $x_{i}$ in the reference configuration, accounting for finite strains. The contravariant components of the Cauchy stress tensor $\sigma^{i j}$ and the Kirchhoff stress tensor $\tau^{i j}$ are related by $\tau^{i j}=\sqrt{G / g} \sigma^{i j}$. The metric tensors in the current and reference configurations are denoted by $G_{i j}$ and $g_{i j}$, with determinants $G$ and $g$, and 
the incremental stress-strain relationship is of the form $\dot{\tau}^{i j}=L^{i j k l} \dot{\eta}_{k l}$, where $L^{i j k l}$ are the instantaneous moduli.

The Lagrangian strain tensor is given by

$$
\eta_{i j}=\frac{1}{2}\left(u_{i, j}+u_{j, i}+u_{, i}^{k} u_{k, j}\right)
$$

where $u^{i}$ are the displacement components on the reference base vectors and ()$_{, j}$ denotes covariant differentiation in the reference frame. Numerical solutions are obtained by a linear incremental solution procedure, based on the principle of virtual work

$$
\int_{V} \tau^{i j} \delta \eta_{i j} d V+\int_{S_{I}}\left\{T_{n} \delta\left(\delta_{n}\right)+T_{t} \delta\left(\delta_{t}\right)\right\} d S=\int_{S} T^{i} \delta u_{i} d A
$$

Here, $V$ and $S$ are the volume and surface of the body in the reference configuration, respectively, $S_{I}$ is the bonded surface cohesive region, and $T^{i}$ are contravariant components of the nominal surface tractions. An incremental version of the PVW (16) is used for the numerical solution. The displacement fields are approximated in terms of planar 8-noded isoparametric elements. The volume integral in equation (16) is carried out by using 2x2 integration points within each element.

The region analyzed numerically is specified by $-a \leq x_{2} \leq a$ and $0 \leq x_{1} \leq h$ in terms of the reference coordinates $x_{i}$ (see Fig.3b). The plunger is pressed against the surface $-a \leq x_{2} \leq-d$ at $x_{1}=0$, with the plunger displacement $\Delta$ in the $x_{1}$-direction. The plate is supported on the back surface $d \leq x_{2} \leq a$ at $x_{1}=h$ with zero displacement in the $x_{1}$-direction. The cohesive plane, on which shear localization and failure is modelled, coincides with the $x_{1}$ axis. In all but two of the simulations, the gap, $d$, between the plunger and the cohesive plane is taken to be zero. Full sticking is assumed at both at the plunger and the support so that zero displacement in the $x_{2}$-direction is prescribed at these surfaces. Even though this is a plane strain analysis, the displacements in the $x_{2}$-direction are prescribed to be zero at the lower edge, $x_{2}=-a$, for $0 \leq x_{1} \leq h$, thus modelling an effect similar to that at the centerline for circular 
geometries such as that in Fig. 1. The surface at $x_{2}=a$ is traction-free. The initial geometry is here taken to be specified by $h / a=0.8$. Fig. 4.a shows the initial mesh in the vicinity of the cohesive interface, with $64 \times 4$ uniform quadrilaterals on each side of the interface. The length of one square element inside this uniformly meshed region is denoted $D_{0}$. To adequately resolve the fracture process, the length of the active process region (i.e. the region where $\lambda_{1}<\lambda<1$ ) should not be smaller than three or four times this element length, $D_{0}$.

Fig. $4 \mathrm{~b}$ shows the deformed mesh at $\Delta / h=0.136$ for the reference case (Case 1 ). This is just before the point where the full cross-section at the interface has attained $\lambda \geq \lambda_{1}$, i.e., the equivalent of localization in the cohesive plane. A numerical difficulty encountered in this case with $d=0$ is that nodal points on the cohesive interface very near the corner of the plunger approach final separation ( $\lambda \geq 1$ ) slowly. A few interface nodal points on the plunger side moved past the plunger corner resulting in significant mesh distortion. This difficulty was resolved by prescribing that no nodal points on the plunger side of the interface can move past the current location of the corner of the plunger. The problem does not arise in analyses where the value of the normalized peak stress $\hat{\tau} / \tau_{Y}$ is sufficiently small, but becomes pronounced when the value of the normalized peak stress is in the upper range considered in this paper.

\section{Trends in Cropping Force and Work}

In this section the results from a selection of simulations will be presented to expose the roles of the parameters identified in Section 2 on the cropping force-displacement behavior and the macroscopic work of cropping. A total of 21 simulations have been carried out. The dimensionless parameters for each simulation are listed in Table 1 and identified by a case number. The four primary parameters are $h / R_{S}, \hat{\tau} / E, N$ and $\tau_{Y} / E$. The thickness of the plate enters only through $h / R_{S}$. An alternative dimensionless parameter to $h / R_{S}$ is

$$
\frac{h \tau_{Y}}{\Gamma_{0}}=\frac{E}{\pi\left(1-v^{2}\right) \tau_{Y}} \frac{h}{R_{S}}
$$

which is also listed in Table 1. 
Case 1 in Table 1 will be used at the reference case throughout this paper. The dimensionless parameters for Case 1 are believed to be representative of a plate of a tough, intermediate strength steel of several millimeters thickness, such as the DH 36 plate discussed in the Introduction. Neither $\hat{\tau} / \tau_{Y}$ nor $h / R_{S}$ are known for this material, or any other comparable material, and thus the choices for these parameters must be regarded as educated guesses. In addition to the parameters listed in Table 1, the other parameters which must be specified in the simulation code are taken as:

$$
v=0.3, \quad \lambda_{1}=0.15, \quad \lambda_{2}=0.5, \quad \delta_{n}^{c} / \delta_{t}^{c}=1
$$

The first three are used in all the simulations. Simulations with values of $\delta_{n}^{c} / \delta_{t}^{c}$ other than 1 have been carried out and will be reported later, but $\delta_{n}^{c} \quad$ (and $\hat{\sigma}=\left(\delta_{t}^{c} / \delta_{n}^{c}\right) \hat{\tau}$ ) have very little effect on the predictions due to the dominance of shearing in the cropping process.

\subsection{The Role of $h / R_{S}$ - Specifically, the Role of the Specific Microscopic Work of} Fracture, $\Gamma_{0}$, and/or the Plate Thickness, $h$. In this set of simulations, $N$ and $\tau_{Y} / E$ are fixed and $h / R_{S}$ is varied for two choices of $\hat{\tau} / \tau_{Y}$. By (17), for each choice of $\hat{\tau} / \tau_{Y}$, varying $h / R_{S}$ is equivalent to varying $h \tau_{Y} / \Gamma_{0}$. In other words, if one considers all the other dimensional parameters as being fixed, this set of simulations is equivalent to either varying only $\Gamma_{0}$ with $h$ fixed or varying $h$ with $\Gamma_{0}$ fixed. For this set of simulations, varying only $\Gamma_{0}$ is accomplished by varying only $\delta_{t}^{c}$ (c.f., (13)). Fig.5 displays the dimensionless forcedisplacement curves. The influence of the parameter $h / R_{S}$ (or, equivalently, $h \tau_{Y} / \Gamma_{0}$ ) on the force-displacement behavior is significant, especially so considering that dimensionless results for any model based only on plastic shearing alone cannot contain a dependence on $h$, at least assuming that there is no gap, $d=0$. This assertion follows from the dimensional argument that the only parameter containing a length dimension available to combine with $h$ to form a dimensionless parameter is $\Gamma_{0}$, e.g., $h / R_{S}$ or $h \tau_{Y} / \Gamma_{0}$.

Values of the two dimensionless measures of the specific cropping work are given in Table 1. While it is essential to make use of dimensionless parameter combinations for problems such as cropping which have a large number of independent parameters, relations among dimensionless 
variables often mask connections between dimensional quantities. This is particularly true for the specific work of cropping. Thus, to clearly display the effect of the specific microscopic work of fracture, $\Gamma_{0}$, on the specific work of cropping, $\Gamma_{C R O P}$, normalized variables are used in Fig. 6 with $\Gamma_{\text {CROP }} /\left(\Gamma_{\text {CROP }}\right)_{R}$ plotted as a function of $\Gamma_{0} /\left(\Gamma_{0}\right)_{R}$ for plates with the same thickness $h$. Here, and subsequently, the subscript $R$ denotes a value from Table 1 for the reference Case 1. These same curves provide the relation between $\Gamma_{C R O P} /\left(\Gamma_{C R O P}\right)_{R}$ and $(h)_{R} / h$ for plates having the same $\Gamma_{0}$. Clearly, the specific microscopic work of fracture has significant influence on the specific macroscopic cropping work, even though $\Gamma_{0}$ is a small fraction of $\Gamma_{\text {CROP }}$ (c.f., Table 1). The quantitative predictions in Fig. 6 back up the earlier modeling of Atkins [1-4], who seems to be the first to view cropping as a shear fracture problem and not just a plastic shear-off process.

The curves in Fig. 6 also reveal the effect of plate thickness on the specific cropping work for the reference material. Doubling the thickness decreases $\Gamma_{\text {CROP }}$ by about $30 \%$, and vice versa. Recall that both $\Gamma_{C R O P}$ and $\Gamma_{0}$ are defined as energy per separated area, with units $\mathrm{Jm}^{-2}$. Thus, the simulations predict that the total work required to crop a plate of the considered material increases by only a factor of about 1.4 when the thickness of the plate is doubled-much less than the factor of 2 expected if $\Gamma_{\text {CROP }}$ were independent of thickness. Conversely, cropping a plate of this material with half the thickness only reduces the total cropping work by a factor of about 0.7 rather than $1 \frac{1}{2}$. This dependency on plate thickness is not at all obvious. However, it follows directly from dimensional arguments that there must be a dependence of $\Gamma_{\text {CROP }}$ on $h$ if there is a dependence on $\Gamma_{0}$-as noted above, the only length parameter in the problem that can be used to form a dimensionless parameter involving $\Gamma_{0}$ is $h$.

The several stages of the cropping process are indicated in Fig. 7 for the reference case. The entire cropping region undergoes extensive plastic yielding through the thickness of the plate prior to the onset of any crack growth. The fully plastic nature of the cropping process, emphasized in the Introduction, is evident in Fig. 7 where large nonlinear displacements due to plasticity occur prior to any cracking. At the maximum load, the shear cracks emerging from the 
corners of the platens have grown to a length of approximately $h / 20$. With further imposition of the cropping displacement, each zone of shear decohesion extends towards the center of the plane until, at $\Delta / h \cong 0.14$, they merge at the center. Prior to this point in the process, significant plastic deformation takes place outside the cohesive zone. However, for $\Delta / h>0.14$, the deformation is mainly confined to the cohesive zone, the cracks grow towards each other, and the cropping force drops dramatically until the cracks connect at the center at $\Delta / h \cong 0.16$.

There are some differences between the sequence of events occurring in Fig. 7 and those laid out in the early work of Johnson and Slater [14]. These authors argued that at the time there paper was written there was no evidence for shear cracking prior to the maximum punch force, and they suggest that the maximum is a result of shear localization. In their view, cracking first occurs after the maximum load is attained. Specific details such as these will depend on the tendency for a given material to undergo shear localization prior to shear damage. To some extent, the sequence of events can be explored within the context of the present model by varying the cohesive zone parameters. For example, decreasing the shear strength, $\hat{\tau}$, while fixing, or increasing, the specific work of fracture, $\Gamma_{0}$, promotes early localization relative to shear cracking. In addition, Johnson and Slater [4] discuss the role friction is expected to play once a shear crack has developed. Friction is not taken into account in the present model and, thus, the cropping force vanishes when the cracks connect.

4.2 The Role of the Normalized Shear Strength, $\hat{\tau} / \tau_{Y}$. In this set of simulations $h / R_{S}, N$ and $\tau_{Y} / E$ are fixed at the reference values and $\hat{\tau} / \tau_{Y}$ is varied. To generate the forcedisplacement curve presented in Fig. 8 and the associated values of the cropping work in Table 1, both $\hat{\tau}$ and $\delta_{t}^{c}$ are varied such that $\Gamma_{0}=0.675 \hat{\tau} \delta_{t}^{c}$ and $h$ are unchanged. The microscopic shear strength, $\hat{\tau}$, obviously has significant effect on both the maximum cropping force and the cropping work. The dimensionless cropping work from Table 1 for these three cases are: $\Gamma_{\text {CROP }} / \Gamma_{0}=4.27,6.11,8.55$ for $\hat{\tau} / \tau_{Y}=2.77,3.12,3.46$, respectively. This strong dependency is similar to the role that the cohesive tensile strength plays in mode I crack growth resistance in small scale yielding [6].

The cropping process is dominated by shear both in the cohesive zone and in the surrounding field of plasticity as the strong dependence on $\hat{\tau}$ reflects. All but one of the simulations listed in 
Table 1 take $\delta_{n}^{c} / \delta_{t}^{c}=1$, which by the cohesive law (9) implies that under a purely normal separation the peak stress would be $\hat{\sigma}=\left(\delta_{t}^{c} / \delta_{n}^{c}\right) \hat{\tau}=\hat{\tau}$. For most materials this would be an unusually low peak for normal separation. To see the effect of the parameter $\delta_{n}^{c} / \delta_{t}^{c}$ on the cropping process, a simulation (Case 21) was carried out with dimensionless parameters identical to those of the reference case except with $\delta_{n}^{c} / \delta_{t}^{c}=1 / 2$ corresponding to $\hat{\sigma}=2 \hat{\tau}$. Both the normalized force-displacement curve and the normalized specific cropping work (see Table 1) were essentially unchanged from the reference Case 1. For the present model, the mixed mode cohesive characteristics as determined by $\delta_{n}^{c} / \delta_{t}^{c}$ have almost no influence on cropping, at least with no gap ( $d / h=0$ ), consistent with the notion that cropping is dominated by shearing.

4.3 The Role of Strain Hardening $N$. The simulated force-displacement curves in Fig. 9 show the effect of varying $N$ with the other dimensionless parameters held fixed. Increased strain hardening elevates the cropping force, but makes it easier to attain the tractions required to cause cohesive shear. The result is that higher strain hardening decreases the normalized displacement, $\Delta / h$, at which the cropping force become zero. With fixed $\tau_{Y}$, the cropping work is also a strong function of $N$, varying from $\Gamma_{C R O P} / \tau_{Y} h=0.0973$ for $N=0.33$ to $\Gamma_{\text {CROP }} / \tau_{Y} h=0.626$ for $N=0.125$ (Table 1). Of course, for a family of alloys, processing treatments that increase $N$ are usually accompanied by a decrease in $\tau_{Y}$, and thus, when that is the case, a change in the parameter $\tau_{Y} / E$ must also be taken into account.

4.4 The Role of $\tau_{Y} / E$. In this subsection, the effect of changes in $\tau_{Y} / E$ with $h / R_{S}, \hat{\tau} / \tau_{Y}$ and $N$ held fixed are examined. To carry out these calculations, $E$ was changed, but to ensure that $h / R_{S}$ did not change, $\Gamma_{0}$ was also changed such that the product $E \Gamma_{0}$ was fixed. The normalized force displacement curves are shown in Fig. 10, where the influence of $\tau_{Y} / E$ is seen to be significant. The role of $\tau_{Y}$ (or $E$ ) is not easy to decipher because it is present in three (two) of the dimensionless parameter combinations. While the dimensionless specific work of cropping, $\Gamma_{C R O P} / \tau_{Y} h$, is strongly affected by variations in $\tau_{Y} / E$, the second measure in Table 1, $\Gamma_{C R O P} / \Gamma_{0}$, is only weakly dependent on $\tau_{Y} / E$. A four-fold change in $\tau_{Y} / E$ (with $h / R_{S}$, 
$\hat{\tau} / \tau_{Y}$ and $N$ fixed) produces only about a $25 \%$ change in $\Gamma_{C R O P} / \Gamma_{0}$. The important conclusion to be drawn is that the cropping work as measured by $\Gamma_{C R O P} / \Gamma_{0}$ depends primarily on $h / R_{S}$, $\hat{\tau} / \tau_{Y}$ and $N$.

4.5 The role of Pre-stress $\sigma_{P S} / \sigma_{Y}$. To illustrate one possible application of the model, the effect of uniform pre-straining [1] is considered. Assume the plate is strained in uniaxial tension beyond yield to a stress $\sigma_{P S}$ then released to the unstressed state prior to cropping. This prestressing is accounted for in the cropping simulations by simply expanding the starting radius of Mises yield surface from its initial value associated with $\sigma_{Y}$ to the value associated with $\sigma_{P S}$. Case 17, with $\sigma_{P S} / \sigma_{Y}=1.2$, and Case 18, with $\sigma_{P S} / \sigma_{Y}=2$, given in Table 1, have their other parameters identical to those of the reference, Case 1. (In Table 1, for all the other cases, $\sigma_{P S} / \sigma_{Y}=1$ has been used to indicate there has been no plasticity due to pre-stress.) The cropping force-displacement curves in Fig. 11 reveal that a small pre-stress, $\sigma_{P S} / \sigma_{Y}=1.2$, has essentially no effect on cropping. However, the larger value, $\sigma_{P S} / \sigma_{Y}=2$, has a noticeable effect on the cropping curve and reduces the specific work of cropping by about $10 \%$.

4.6 The Role of the Gap $d / h$. A few computations have been carried out to study the effect of a cropping gap. Specifically, for Case 19, the initial gap between the edge of the plunger and the cohesive plane was taken to be two element sizes, $d=2 D_{0}$, and the same gap is assumed at the edge of the supporting tool. Thus, the cohesive plane coincides with the center of the gap. If the mesh in Fig. 4a is used, strong mesh distortion at the edge of the plunger leads to early break down of the computation. Therefore, a specially refined mesh is used around the point where intense straining is induced at the edge of the plunger, as shown in Fig. 12a. This enables the computation to proceed, but the material in the gap rotates significantly, even to the extent that it overlaps the material under the plunger. Consequently, an extra condition is introduced stipulating that the material in the gap cannot penetrate the plunger, as illustrated by the deformed mesh in Fig. 12b. This contact condition is taken to be frictionless sliding.

The normalized force-displacement curves for two gaps sizes, $d / h=0.031$ and 0.047 , are presented in Fig. 13 along with that for the reference case (Case 1) which has no gap but 
otherwise identical parameters. A gap has a significant influence resulting in larger plastic deformation accompanying the cropping process. For the smaller of the two gap sizes, $d / h=0.031$, the cropping work is approximately twice that of the reference case (c.f., Table 1).

As indicated by the above discussion, the large plastic strain and extensive rotation accompanying the shear decohesion process is challenging to simulate, especially when a gap is present. More extensive studies accounting for the gap are clearly required. Handbook rules of recommending maximum gap to thickness ratios for cropping and modeling these is part of the challenge ahead. It should also be mentioned that the effect of changing the relative tensile strength in the cohesive zone, $\hat{\sigma}=\left(\delta_{t}^{c} / \delta_{n}^{c}\right) \hat{\tau}$, has not been studied in the presence of a gap when it is more likely to be important [5].

\section{Concluding Remarks}

Cropping falls within the larger field concerned with the mechanics and physics of cutting [18]. The present paper has introduced a model which links macroscopic aspects of cropping, such as the cropping force-displacement behavior and the work of cropping, to properties at the microscopic scale such as the fundamental fracture energy of the material and its shear strength. Predictions based on the model have been presented to reveal trends in this relationship. In the examples presented in the body of the paper, the specific macroscopic work of cropping ranges from 2 to 15 times the specific microscopic work of fracture. Nevertheless, even when the microscopic work of fracture comprises only a small fraction of the total work, it has been shown to play a critical role in establishing the macroscopic behavior. The present cropping study parallels earlier efforts to predict the macroscopic mode I fracture toughness of ductile metal alloys in terms of more fundamental microscopic material properties [6]. In so doing, the present study advances the case made by Atkins [1-4] that cropping should be viewed as a fracture problem. In the terminology of nonlinear fracture mechanics, cropping is a large scale yielding fracture problem. Cropping presents its own special challenges owing to the large plastic strains that inevitably accompany the process and the fact that shear localization and shear fracture are less well understood in terms of fundamental material mechanisms than the tensile fracture of ductile metals under high stress triaxiality. The challenges are both computational and physical. 
As mentioned in connection with the introduction of the reference case, the choices of the peak shear strength and work of fracture characterizing the cohesive zone have not been directly calibrated with experiments. For example, it is possible that the microscopic parameters of the steel, DH36, lie outside the range covered by the simulations in this paper. The fact that the plunger displacement at failure, $\Delta / h \cong 0.5$, for the DH36 test in Fig. 1b is larger than any of the values found in the present simulations suggests this might be the case. The main purpose of the present paper has been to expose trends in the roles of the parameters controlling cropping. Future efforts will be undertaken to make direct comparisons with cropping experiments. Finally, it must be re-emphasized that important factors could be added to the present model which have not be taken into account, such as tool deformation, friction, and material temperature and rate-dependence.

\section{References}

[1] Atkins, A.G., “On cropping and related processes,”Int. J. Mech. Sci., 22, 1980, pp. 215-231.

[2] Atkins, A.G., “Surfaces produced by guillotining,” Phil. Mag., A43, 1981, pp. 627-641.

[3] Atkins, A.G., “On the mechanics of guillotining ductile metals,” J. Mat. Proc. Tech., 24, 1990, pp. 245-247.

[4] Atkins, T., "Perforation of metal plates due to through-thickness shearing and cracking. Optimum toughness/strength ratios, deformation transitions and scaling," Int. J. Impact Eng., 48, 2012, pp. 4-14.

[5] Zhou, Q., Wierzbicki, T., “A tension zone model of blanking and tearing of ductile metal plates,” Int. J. Mech. Sci., 38, 1996, pp. 303-324.

[6] Tvergaard, V. and Hutchinson, J.W., "The relation between crack growth resistance and fracture process parameters in elastic-plastic solids,” J. Mech. Phys. Solids, 40, 1992, pp. 1377-1397.

[7] Tvergaard, V. and Hutchinson, J.W., “The influence of plasticity on mixed mode interface toughness,” J. Mech. Phys. Solids, 41, 1993, pp. 1119-1135.

[8] Borvik, T., Langseth, M., Hoppenstad, O.S. and Malo, K.A., "Ballistic penetration of steel plates,” Int. J. Impact Eng., 22, 1999, pp. 855-886.

[9] Borvik, T., Hoppenstad, O.S., Berstad, T. and Langseth, M., "Perforation of 12mm steel plates by $20 \mathrm{~mm}$ diameter projectiles with flat, hemispherical and conical noses: part II: numerical simulations,” Int. J. Impact Eng., 27, 2002, pp. 37-64. 
[10] Nahshon, K., Pontin, M.G., Evans, A.G., Hutchinson, J.W. and Zok, F.W., "Dynamic shear rupture of steel plates,” J. Mech. Mater. Struct., 2, 2007, pp, 2049-2065.

[11] Xue, Z., Pontin, M.A., Zok, F.W. and J.W. Hutchinson, J.W., "Calibration Procedures for a Computational Model of Ductile Fracture," Engineering Fracture Mechanics, 77, 2010, pp. 492-509.

[12] Gurson, A.L., "Continuum theory of ductile rupture by void nucleation and growth - I. Yield criteria and flow rules for porous ductile media,” J. Engng. Materials Technol, 99, 1977, pp. 2-15.

[13] Nahshon, K., Hutchinson, J., "Modification of the Gurson model for shear failure," European J. of Mech./A Solids, 27, 2008, pp. 1-17.

[14] Johnson, W. and Slater, R.A.C., "Survey of slow and fast blanking of metals at ambient and high temperatures," Proc. Int. Conf. "Manufacturing Technology”. CIRP-ASTME, 1987, pp. 825-851.

[15] Tvergaard, V., "Behaviour of voids in a shear field," Int. J. Fracture, 158, 2009, pp. 41-49.

[16] Tvergaard, V., Nielsen, K.L., "Relations between a micro-mechanical model and a damage model for ductile failure in shear,” J. Mech. Phys. Solids, 58, 2010, pp. 1243-1252.

[17] Hutchinson, J.W., "Finite strain analysis of elastic-plastic solids and structures," In: Hartung, R.F. (Ed.), Numerical Solution of Nonlinear Structural Problems. ASME, New York, 1973, pp. 17-29.

[18] Atkins, T. "The Science and Engineering of Cutting,” Butterworth-Heinemann, 2009. ISBN 075068531X. 


\begin{tabular}{|l|l|l|l|l|l|l|l|l|l|}
\hline Case & $\frac{h}{R_{s}}$ & $\frac{\hat{\tau}}{\tau_{\mathrm{Y}}}$ & & \multicolumn{1}{|c|}{$\frac{\tau_{\mathrm{Y}}}{E}$} & $\frac{d}{h}$ & $\frac{\sigma_{P S}}{\sigma_{Y}}$ & $\frac{h \tau_{Y}}{\Gamma_{0}} *$ & $\frac{\Gamma_{\text {CROP }}}{\Gamma_{0}}$ & $\frac{\Gamma_{\text {CROP }}}{\tau_{\mathrm{Y}} h}$ \\
\hline $1(\mathrm{R})$ & 0.0427 & 3.12 & 0.185 & 0.000785 & 0 & 1 & 19.0 & 6.11 & 0.321 \\
\hline 2 & 0.0427 & 3.12 & 0.185 & 0.000393 & 0 & 1 & 38.0 & 6.59 & 0.173 \\
\hline 3 & 0.0427 & 3.12 & 0.185 & 0.001570 & 0 & 1 & 9.51 & 5.07 & 0.533 \\
\hline 4 & 0.0427 & 3.12 & 0.125 & 0.000785 & 0 & 1 & 19.0 & 11.90 & 0.626 \\
\hline 5 & 0.0427 & 3.12 & 0.156 & 0.000785 & 0 & 1 & 19.0 & 9.46 & 0.498 \\
\hline 6 & 0.0427 & 3.12 & 0.227 & 0.000785 & 0 & 1 & 19.0 & 3.69 & 0.194 \\
\hline 7 & 0.0427 & 3.12 & 0.33 & 0.000785 & 0 & 1 & 19.0 & 1.85 & 0.0973 \\
\hline 8 & 0.0480 & 2.77 & 0.185 & 0.000785 & 0 & 1 & 21.4 & 4.53 & 0.212 \\
\hline 9 & 0.0384 & 3.46 & 0.185 & 0.000785 & 0 & 1 & 17.1 & 7.99 & 0.467 \\
\hline 10 & 0.0427 & 2.77 & 0.185 & 0.000785 & 0 & 1 & 19.0 & 4.27 & 0.224 \\
\hline 11 & 0.0427 & 3.46 & 0.185 & 0.000785 & 0 & 1 & 19.0 & 8.55 & 0.450 \\
\hline 12 & 0.0854 & 3.12 & 0.185 & 0.000785 & 0 & 1 & 38.0 & 9.02 & 0.237 \\
\hline 13 & 0.0284 & 3.12 & 0.185 & 0.000785 & 0 & 1 & 12.7 & 4.91 & 0.387 \\
\hline 14 & 0.0214 & 3.12 & 0.185 & 0.000785 & 0 & 1 & 9.51 & 4.08 & 0.429 \\
\hline 15 & 0.0256 & 3.46 & 0.185 & 0.000785 & 0 & 1 & 11.4 & 5.99 & 0.526 \\
\hline 16 & 0.0192 & 3.46 & 0.185 & 0.000785 & 0 & 1 & 8.56 & 4.94 & 0.578 \\
\hline 17 & 0.0427 & 3.12 & 0.185 & 0.000785 & 0 & 1.2 & 19.0 & 6.08 & 0.320 \\
\hline 18 & 0.0427 & 3.12 & 0.185 & 0.000785 & 0 & 2.0 & 19.0 & 5.55 & 0.292 \\
\hline 19 & 0.0427 & 3.12 & 0.185 & 0.000785 & 0.031 & 1 & 19.0 & 11.96 & 0.630 \\
\hline 20 & 0.0427 & 3.12 & 0.185 & 0.000785 & 0.047 & 1 & 19.0 & 14.20 & 0.747 \\
\hline 21 & 0.0427 & 3.12 & 0.185 & 0.000785 & 0 & 1 & 19.0 & 6.01 & 0.316 \\
\hline
\end{tabular}

In all calculations: $v=0.3, \lambda_{1}=0.15, \lambda_{2}=0.50$ and $\delta_{n}^{c} / \delta_{t}^{c}=1$ (except for \#21, $\delta_{n}^{c} / \delta_{t}^{c}=1 / 2$ ).

Case 1 is the reference case and denoted by a subscript $R$ in the text and figures.

$* h \tau_{Y} / \Gamma_{0}$, given by (17), is not an independent parameter.

Table 1 Simulation Cases. 

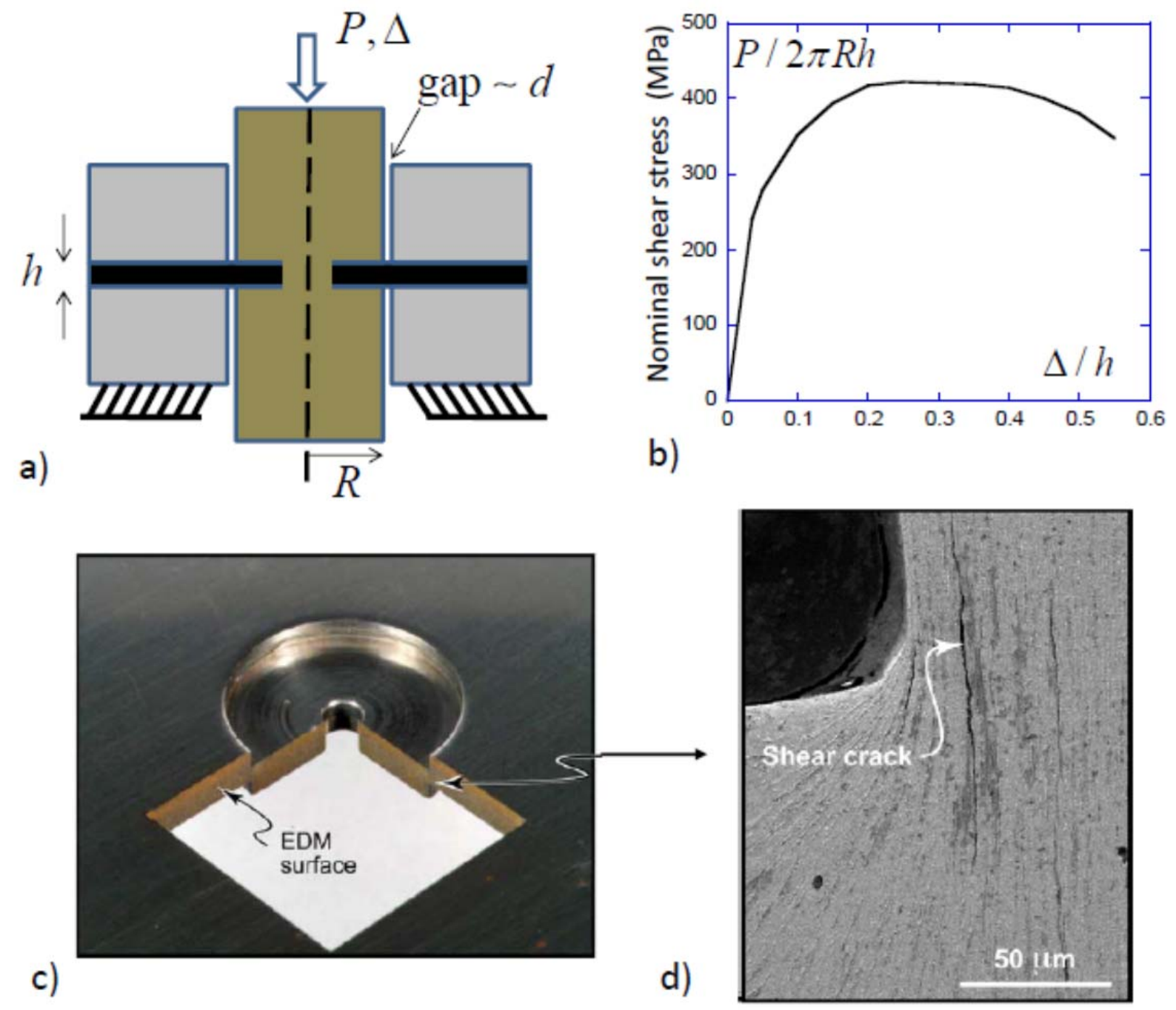

Fig. 1 A cropping shear-off test of Xue, et al.(2010). a) Schematic of the axisymmetric test configuration. The two sections of central plunger are bolted together through the center of the plate whose thickness is $h$. b) An experimentally measured nominal shear stress, $P /(2 \pi R h)$, versus normalized displacement of the plunger, $\Delta / h$, for the steel, DH 36 . For this test: $h=3 \mathrm{~mm}, R=19.05 \mathrm{~mm}$ and the gap width is $d \cong 0.075 \mathrm{~mm}$. Beyond $\Delta / h=0.55$, the curve drops precipitously. c) A test interrupted at $\Delta / h=0.5$ with a section removed from the plate to reveal the details of the shear-off process. d) A blow up of the region at the corner of the plunger showing that shear cracks have formed, some of which are offset from the corner. 

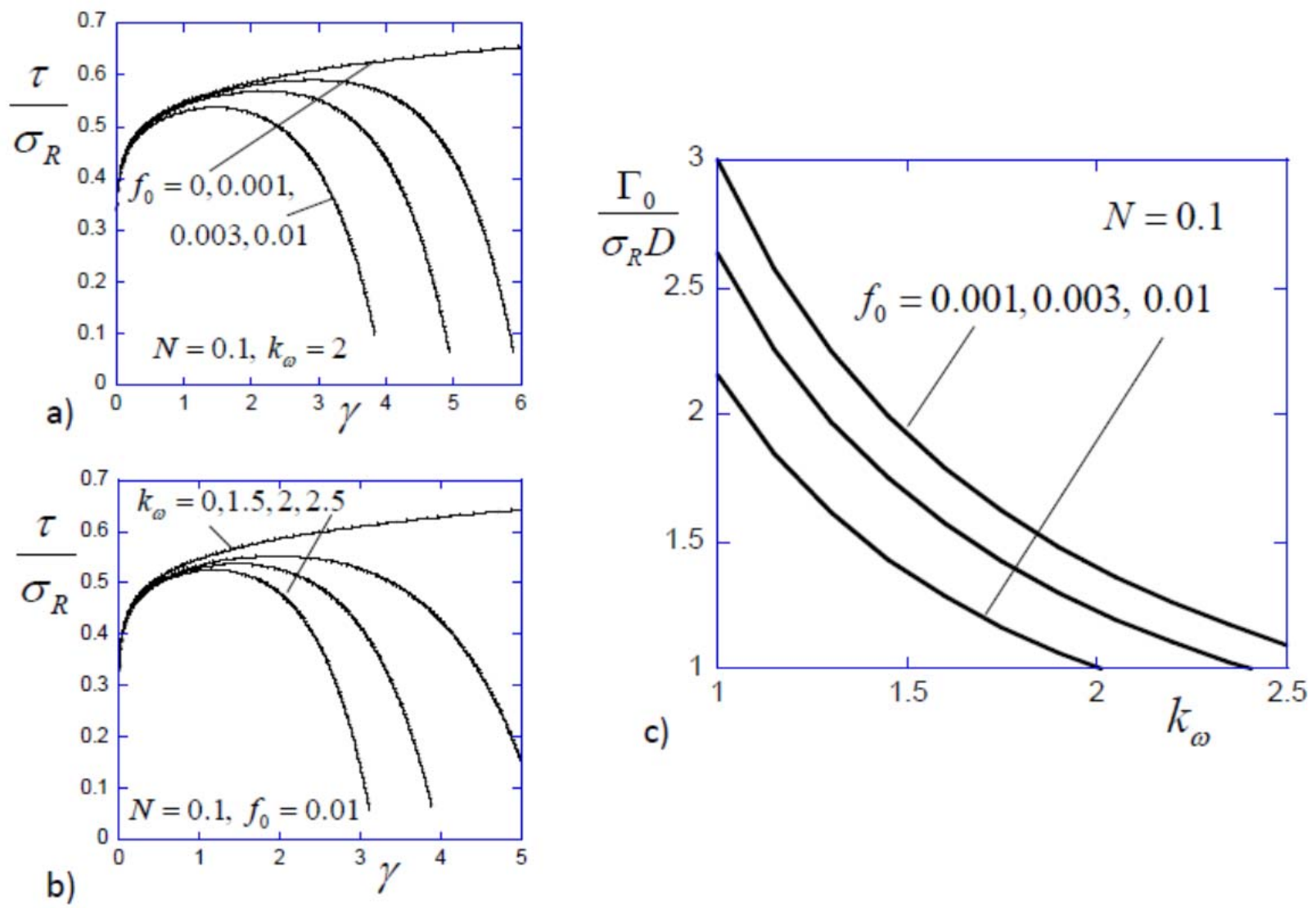

c)

Fig. 2 a) and b): True shear stress versus log shear strain predicted by the extended Gurson model as dependent on the initial effective void volume fraction, $f_{0}$, and shear damage coefficient, $k_{\omega}$. The reference stress, $\sigma_{R}$, is defined in the text. C) The normalized specific work of shear separation, $\Gamma_{0} / \sigma_{R} D$, which is computed for shearing beyond the peak shear stress assuming shearing takes place in a layer of thickness $D$. For this model, the normalized work is only weakly dependent on the strain hardening exponent, $N$. 


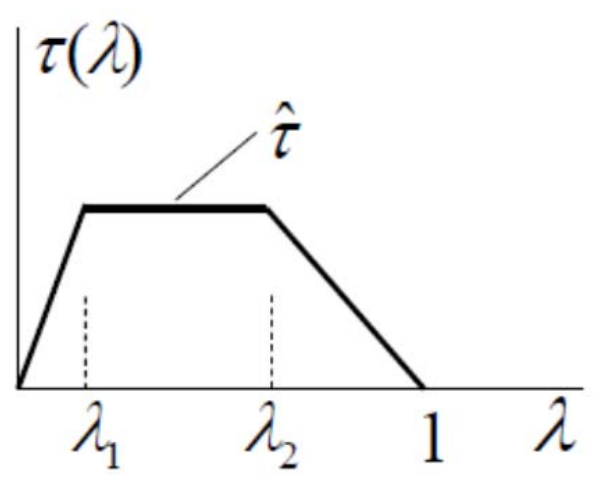

a)

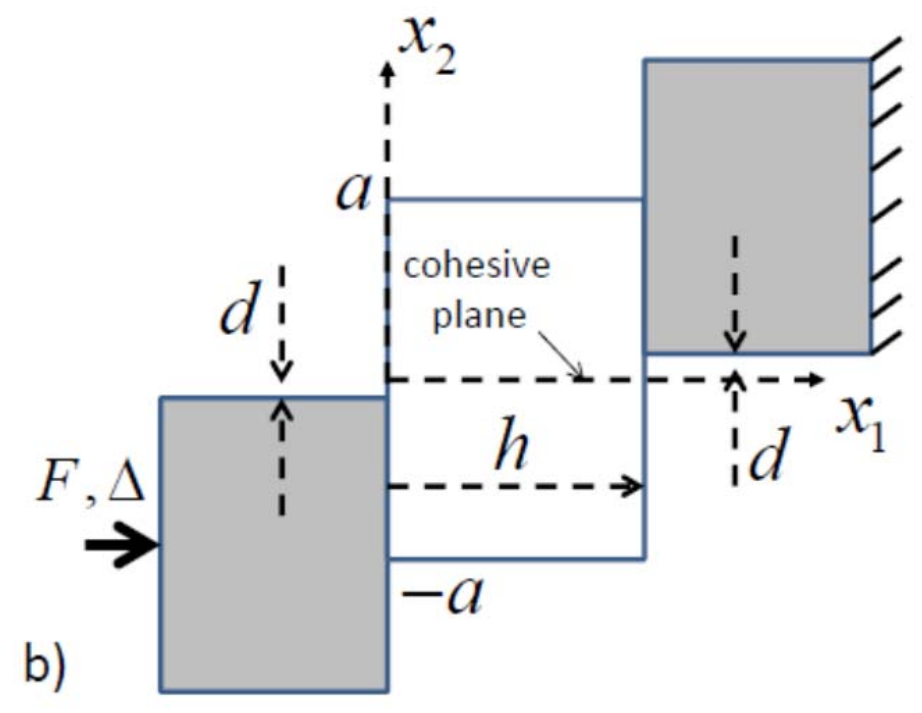

Fig. 3 a) The cohesive traction-displacement relation in shear. b) The undeformed geometry of the numerical model showing the reference coordinates. The cohesive plane in the undeformed state lies along the $x_{1}$ axis. The bottom surface along $x_{2}=-a$ is constrained to undergo frictionless sliding with zero vertical displacement. The upper surface along $x_{2}=a$ is tractionfree. Sliding is suppressed at the contact surfaces between the rigid tools and the metal block. 

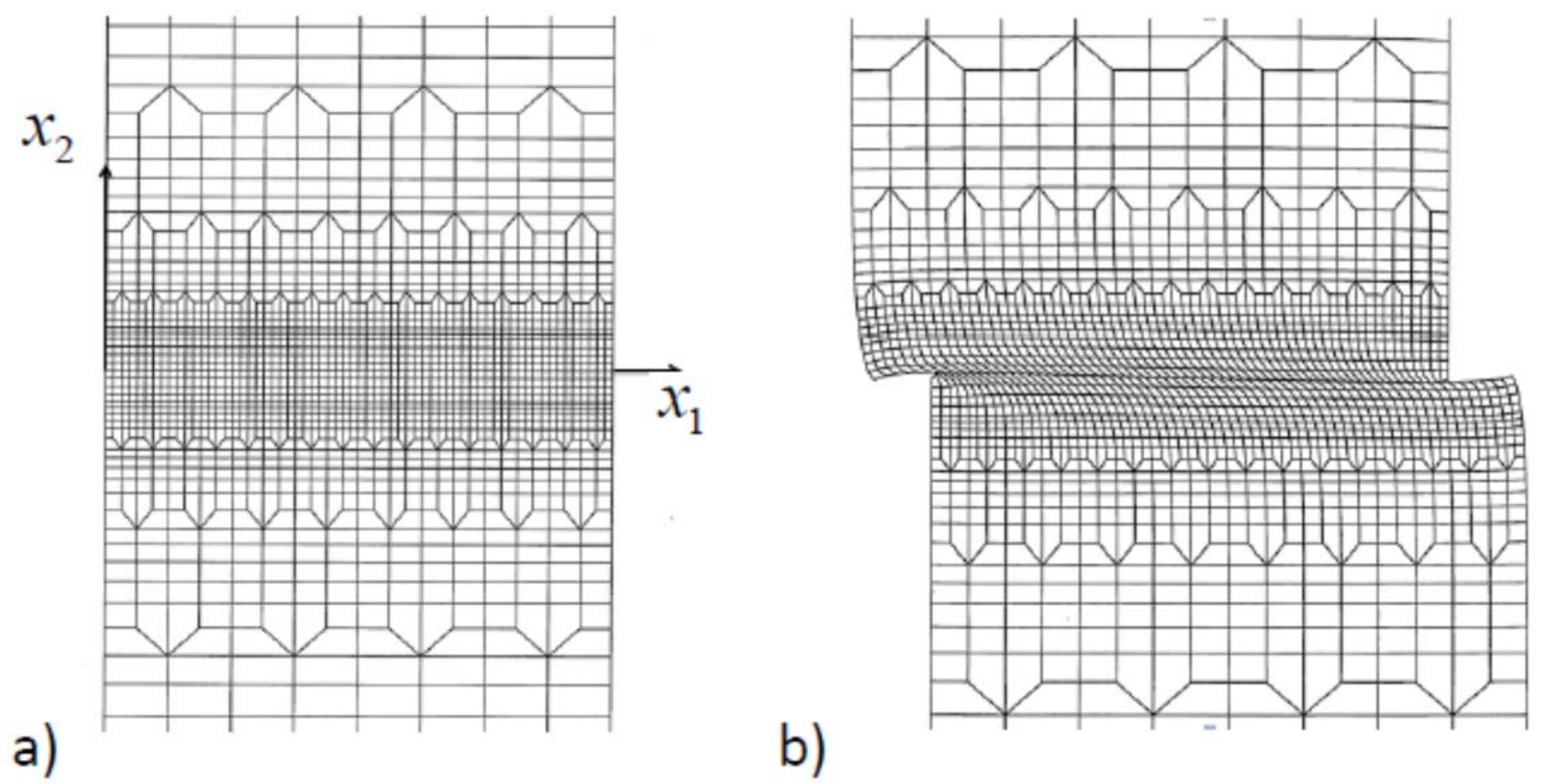

Fig. 4 a) The mesh in the undeformed state. The cohesive plane lies along the $x_{1}$-axis. b) The deformed mesh for the reference case (Case 1) at $\Delta / h=0.136$. 


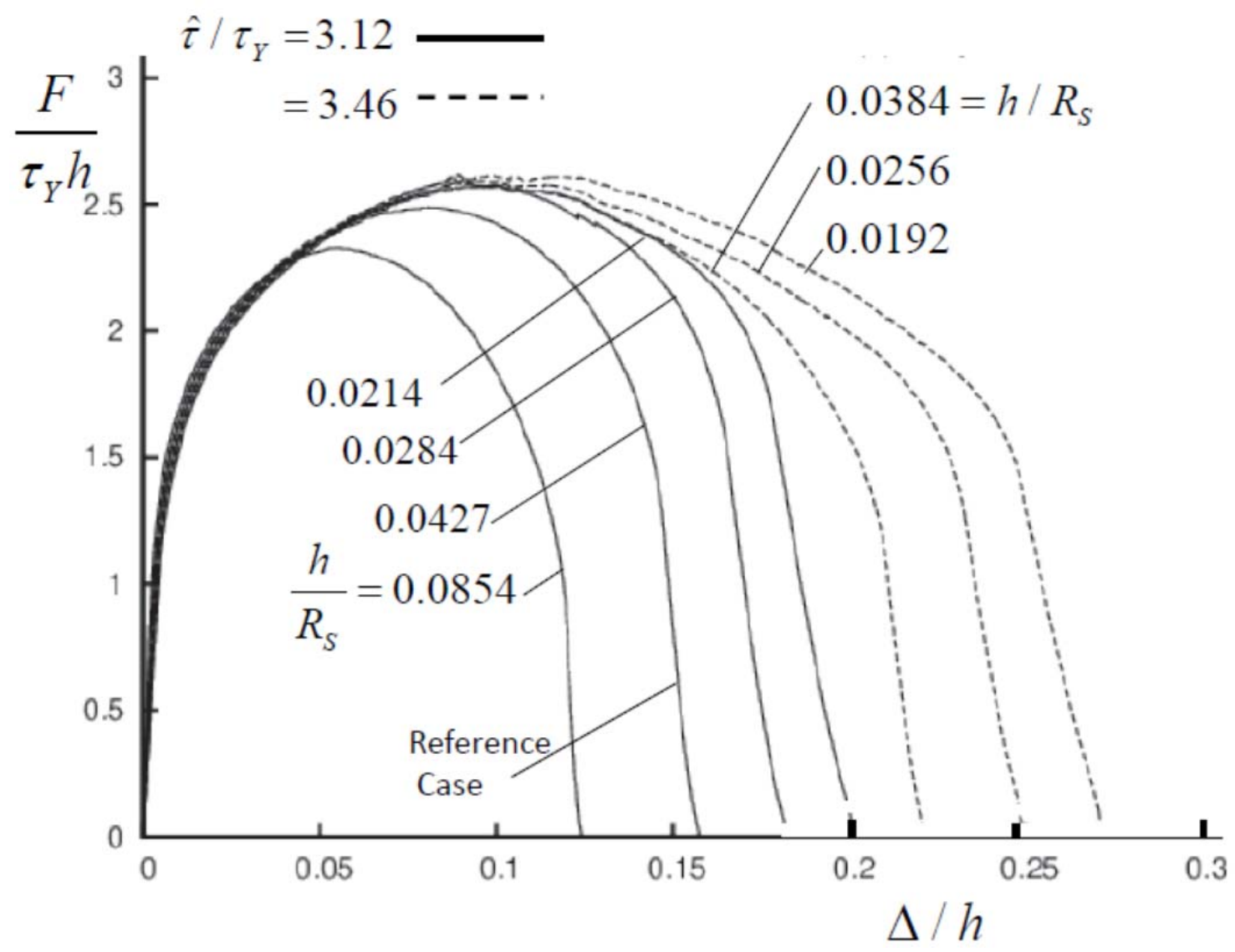

Fig. 5 The role of $h / R_{S}=\pi\left(1-v^{2}\right) h \tau_{Y}^{2} /\left(E \Gamma_{0}\right)$ on the normalized force-displacement relation for cropping for two values of $\hat{\tau} / \tau_{Y}$ and with $N=0.185$ and $\tau_{Y} / E=0.000785$. With all the other dimensional parameters fixed, each set of curves (for a given $\hat{\tau} / \tau_{\mathrm{Y}}$ ) can be interpreted as either the effect of varying $\Gamma_{0}$ (with $h$ fixed) or varying $h$ (with $\Gamma_{0}$ fixed). Cases 1,11,12,13, $14,15,16$. 


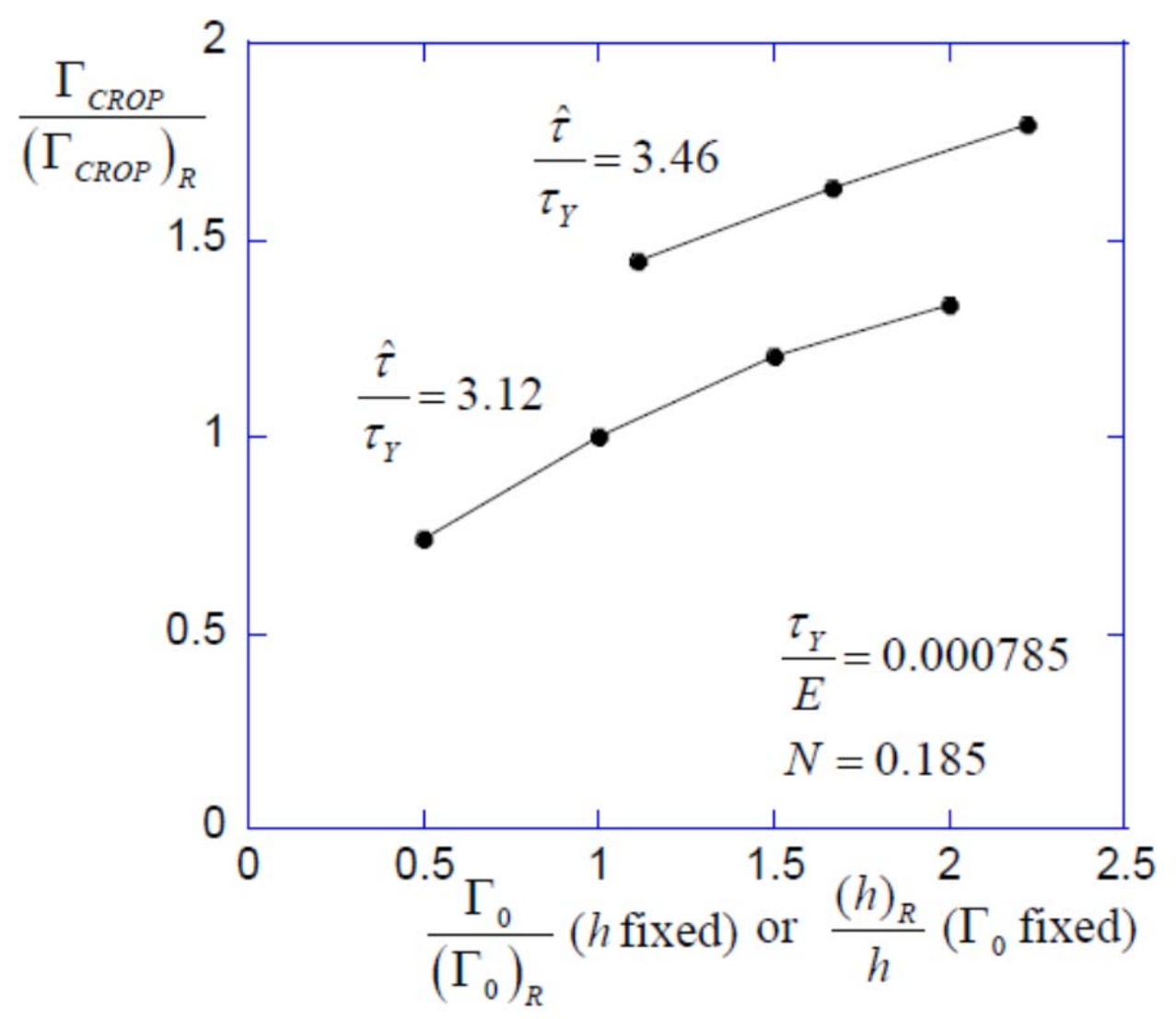

Fig. 6 The dependence of the specific cropping work, $\Gamma_{C R O P}$, on the microscopic specific work of fracture, $\Gamma_{0}$, for plates of the same thickness. Equivalently, the dependence of the specific cropping work, $\Gamma_{C R O P}$, on inverse thickness, $1 / h$, for plates with fixed material properties. The normalizing quantities denoted by the subscript $R$ refer to reference Case 1 . The points plotted are Cases 1, 11, 12, 13, 14, 15, 16. 


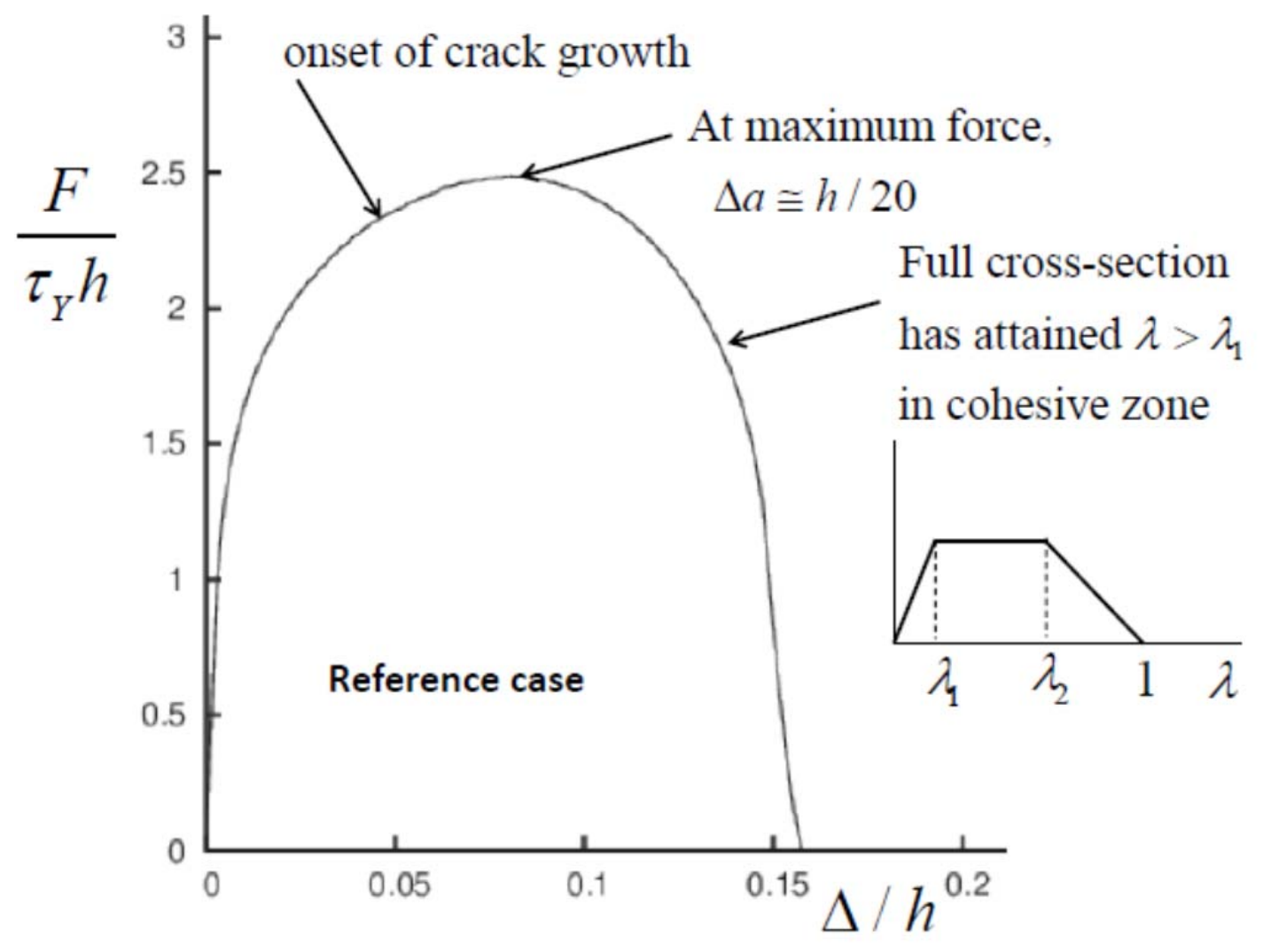

Fig. 7 Three stages of the cropping process illustrated for the reference case. (i) Onset of cracking when $\lambda$ first attains 1 in the cohesive zones at the corners of the cropping tool. (ii) At maximum force the cracks at each corner of the tool on opposite surfaces have extended a distance approximately $1 / 20$ the plate thickness. (iii) The point at which the traction plateau is attained in the cohesive zone throughout the central cross-section of the plate, i.e., $\lambda \geq \lambda_{1}$. As $\Delta$ increases beyond this point, localized shear-off is confined to the cohesive zone and the cracks spread toward the center until they meet at $\Delta / h \cong 0.16$. Case 1 . 


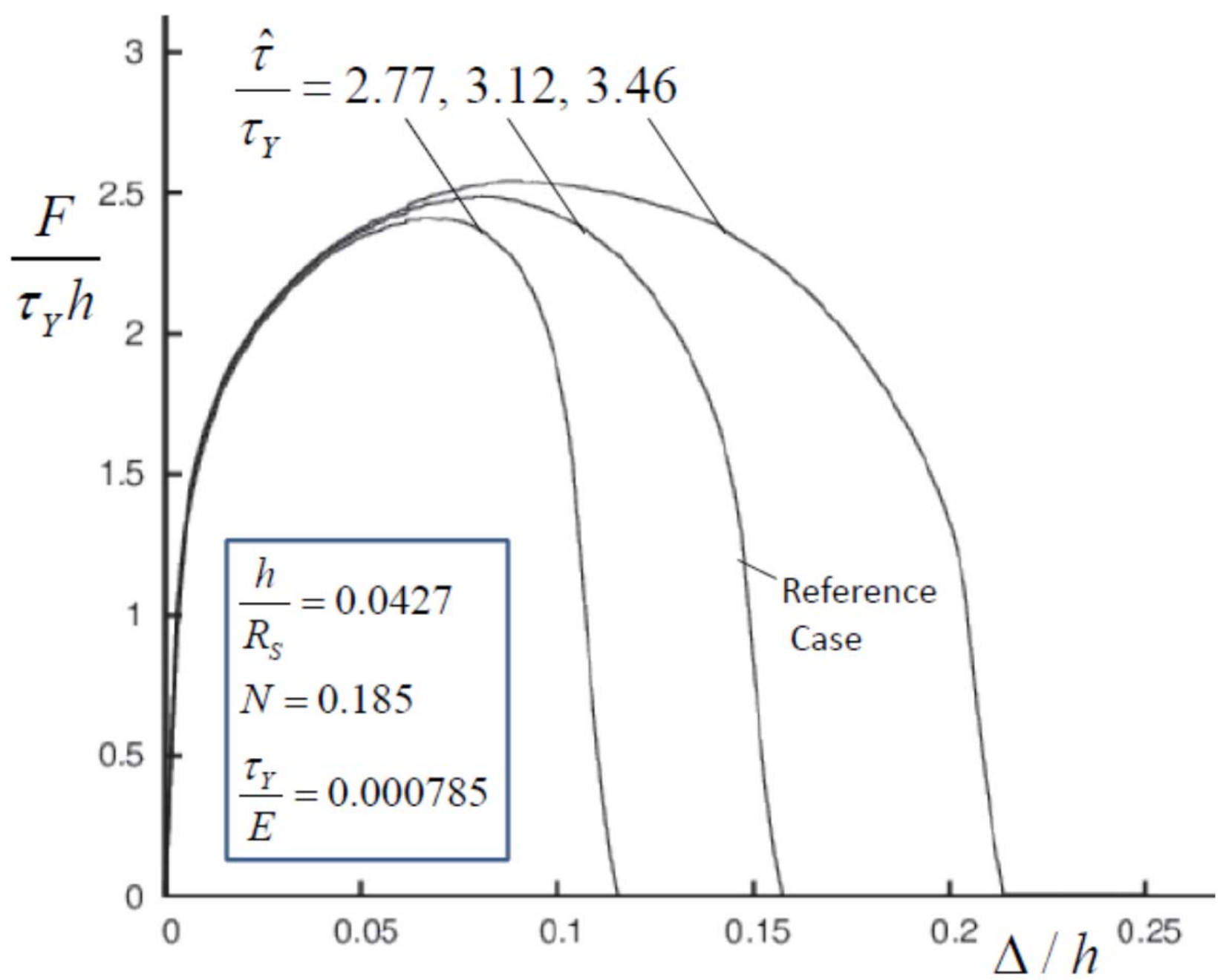

Fig. 8 The role of the normalized shear strength in the cohesive zone, $\hat{\tau} / \tau_{Y}$, on the normalized force-displacement curve with the other dimensionless parameters held fixed. Cases 1,10,11. 


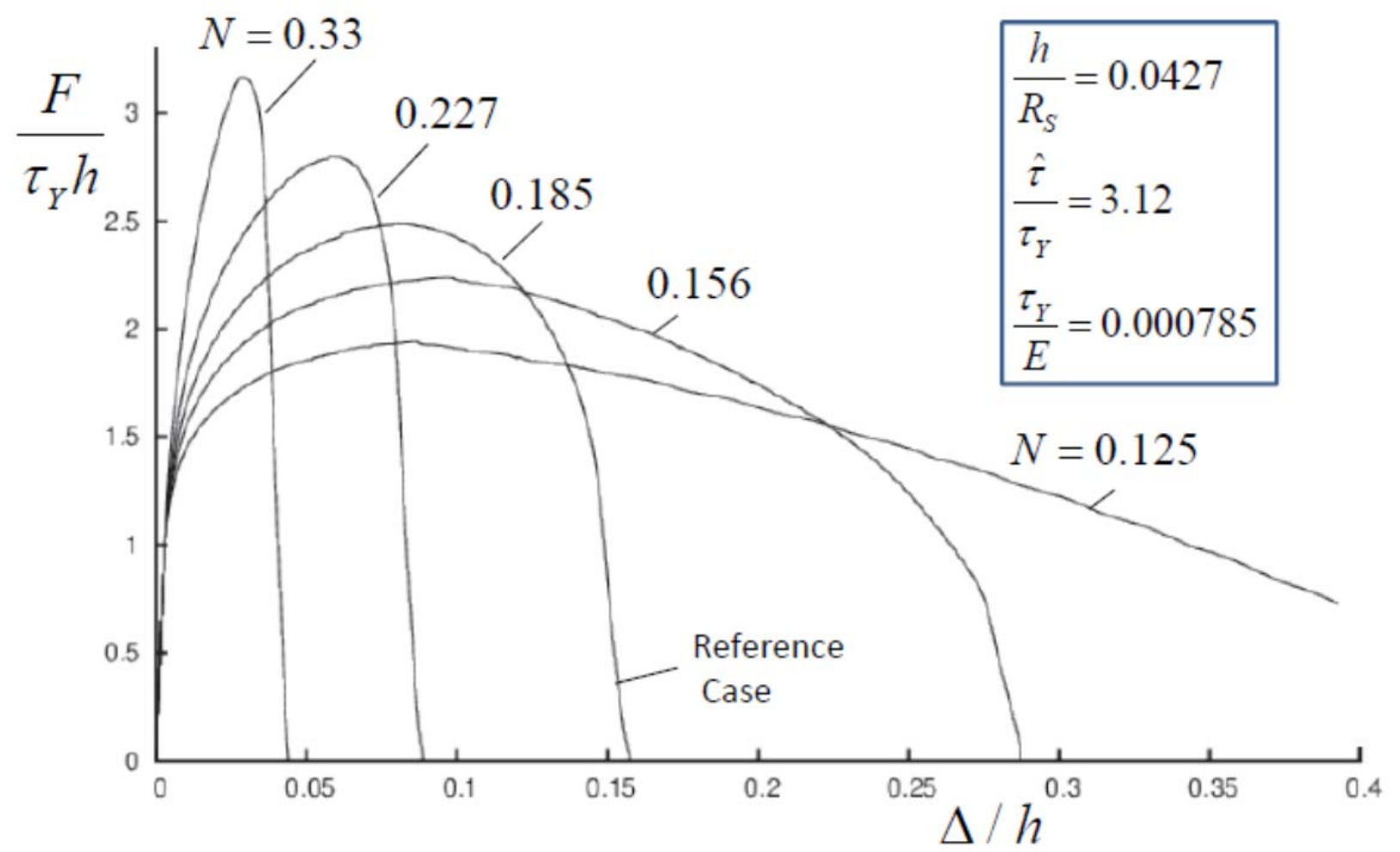

Fig. 9 The role of the strain hardening exponent on the normalized force-displacement curves with the other dimensionless parameters held fixed. Cases 1, 4, 5, 6, 7. 


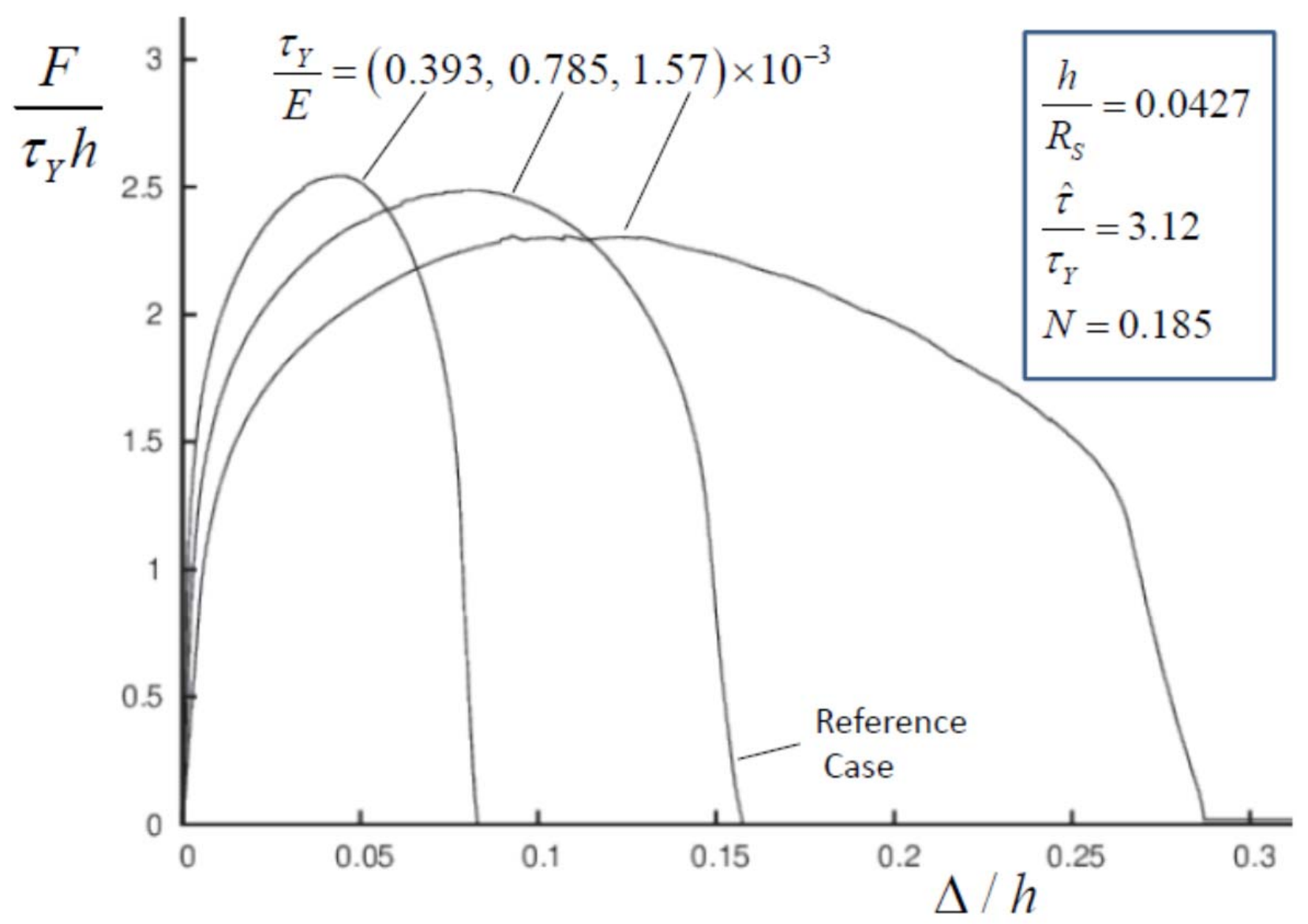

Fig. 10 The role of $\tau_{Y} / E$ on the normalized force-displacement curves with the other dimensionless parameters held fixed. As discussed in the text, the specific cropping work with the normalization, $\Gamma_{C R O P} / \Gamma_{0}$, is only weakly dependent on $\tau_{Y} / E$ with the other dimensionless parameters held fixed. Cases: 1,2, 3. 


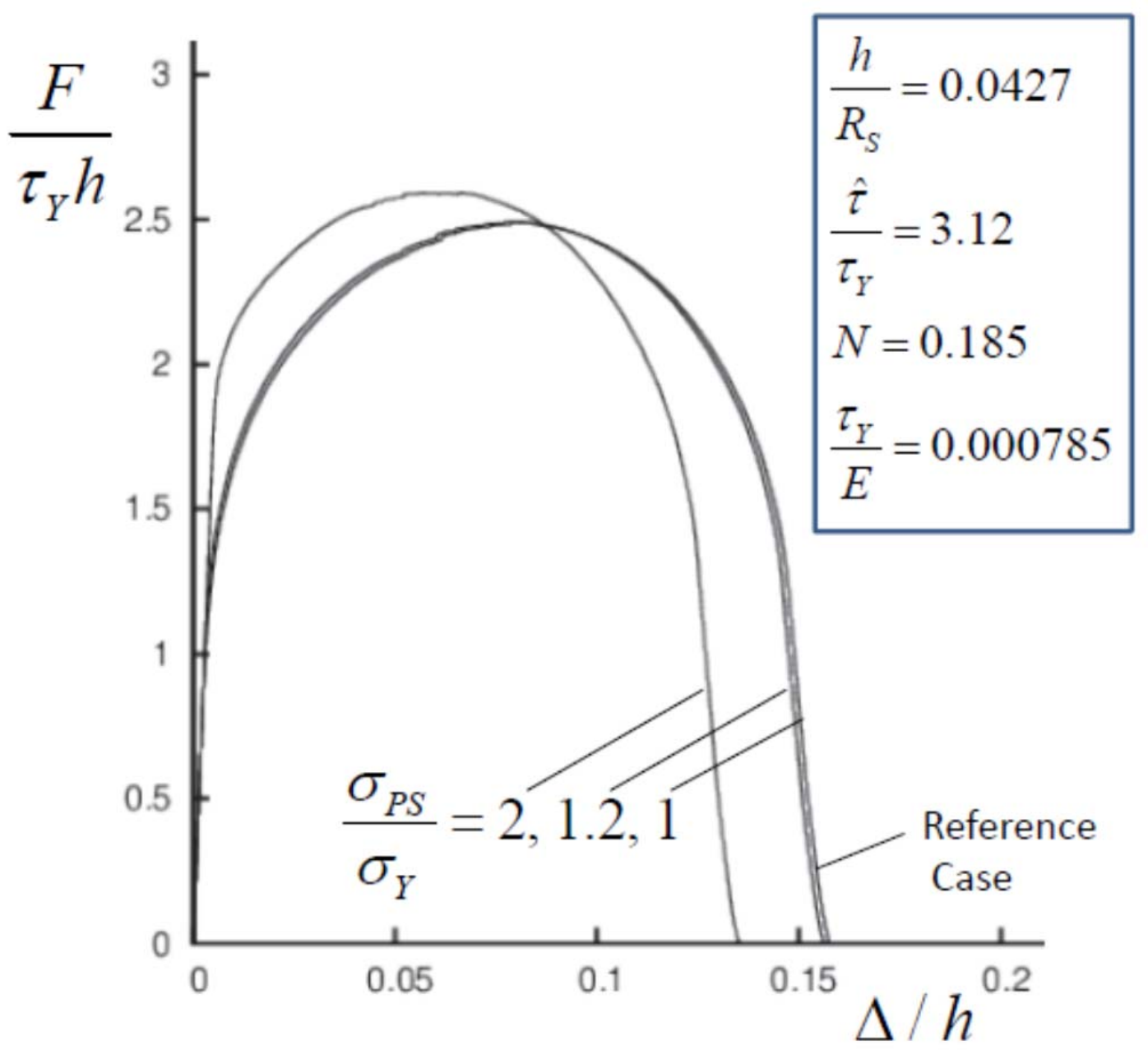

Fig. 11 A tensile pre-stress $\sigma_{P S}$ is applied to the plate to produce uniform plastic stretch and then released prior to cropping. The other dimensionless parameters for the pre-stress cases, (Cases 17, 18), are the same as for Case 1, the reference case. 


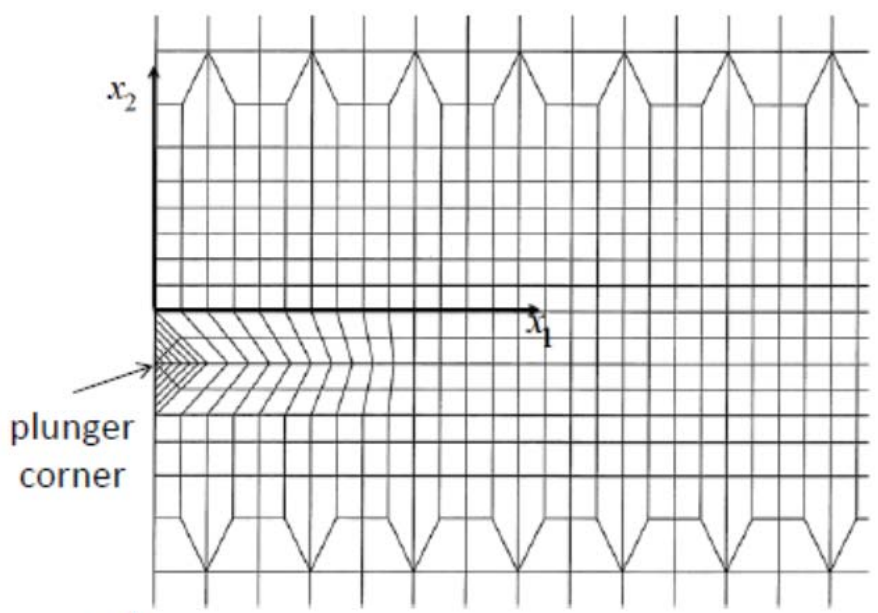

a)

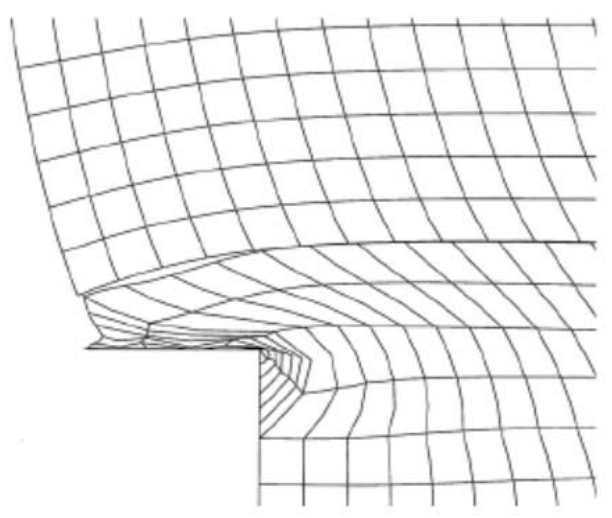

b)

Fig. 12 a) Undeformed mesh for simulation with a gap, $d=2 D_{0}$. b) Deformed mesh with gap in the vicinity of the plunger corner for Case 19 at $\Delta / h=0.108$. 


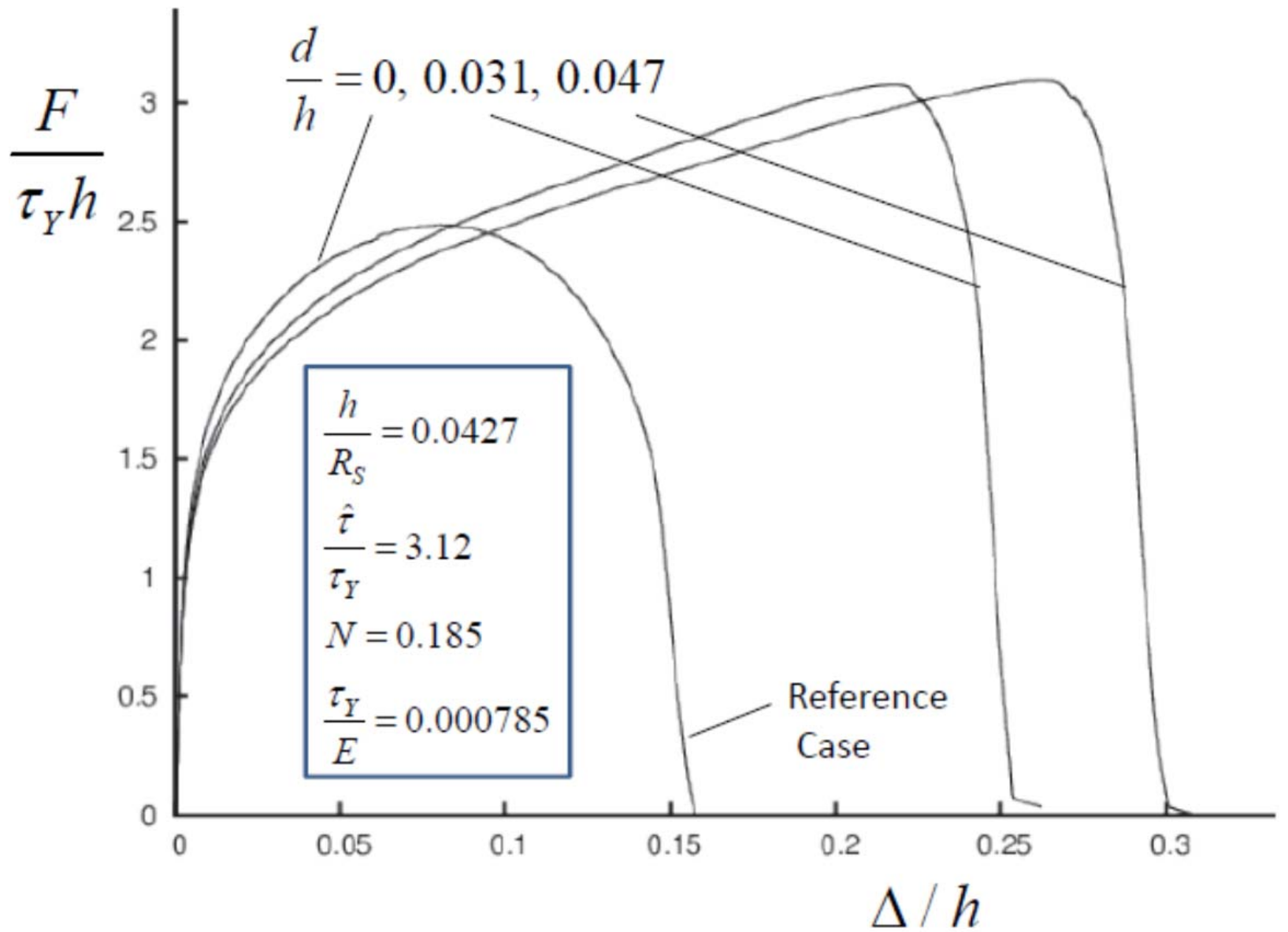

Fig. 13 The role of a gap between the tool corners and the cohesive plane, $d / h$, on the normalized force-displacement curves with the other dimensionless parameters held fixed and equal to those of the reference case. Cases: 1, 19, 20. 